\title{
Conditioned media from human macrophages of M1 phenotype attenuate the cytotoxic effect of 5-fluorouracil on the HT-29 colon cancer cell line
}

\author{
ALEXANDER HEDBRANT $^{1}$, ANN ERLANDSSON ${ }^{1}$, DICK DELBRO $^{2}$ and JONNY WIJKANDER ${ }^{1}$ \\ ${ }^{1}$ Department of Health Sciences, Karlstad University, Karlstad; \\ ${ }^{2}$ School of Health and Medical Sciences, Örebro University, Örebro, Sweden
}

Received June 27, 2014; Accepted August 12, 2014

DOI: $10.3892 / \mathrm{ijo} .2014 .2696$

\begin{abstract}
Resistance of tumor cells to chemotherapy, such as 5-fluorouracil (5-FU), is an obstacle for successful treatment of cancer. As a follow-up of a previous study we have investigated the effect of conditioned media (CM) from macrophages of M1 or M2 phenotypes on 5-FU cytotoxicity on the colon cancer cell lines HT-29 and CACO-2. HT-29 cells, but not CACO-2 cells, having been treated with a combination of M1 CM and 5-FU recovered their cell growth to a much larger extent compared to cells having been treated with 5-FU alone when further cultured for 7 days in fresh media. M1 CM treatment of HT-29, but not CACO-2 cells, induced cell cycle arrest in the $\mathrm{G}_{0} / \mathrm{G}_{1}$ and $\mathrm{G}_{2} / \mathrm{M}$ phases. 5-FU treatment induced accumulation of cells in S-phase in both HT-29 and CACO-2 cells. This accumulation of cells in S-phase was attenuated by combined M1 CM and 5-FU treatment in HT-29 cells, but not in CACO-2 cells. The mRNA expression of cell cycle regulatory proteins and 5-FU metabolic enzymes were analyzed in an attempt to find possible mechanisms for the M1 CM induced attenuation of 5-FU cytotoxicity in HT-29. Thymidylate synthetase (TS) and thymidine phosphorylase (TP) were
\end{abstract}

Correspondence to: Dr Jonny Wijkander, Department of Health Sciences, Karlstad University, Universitetsgatan 2, S-651 88 Karlstad, Sweden

E-mail: jonny.wijkander@kau.se

Abbreviations: 5-FU, 5-fluorouracil; CCN, cyclin; CDC, cell division cycle; CDK, cyclin-dependent kinase; CHK, checkpoint division cycle; CM, conditioned media; CRC, colorectal cancer; DPD, dihydropyrimidine dehydrogenase; FdUMP, fluorodeoxyuridine monophosphate; FOXO, forkhead box O; FUTP, fluorouridine triphosphate; GADD, growth arrest and DNA-damage-inducible; MTHF, methylenetetrahydrofolate; MTHFR, methylenetetrahydrofolate reductase; TAM, tumor-associated macrophages; TP, thymidine phosphorylase; TS, thymidylate synthetase; UMPS, uridine monophosphate synthetase

Key words: chemotherapy, 5-fluorouracil, colon cancer cell line, HT-29, CACO-2, M1 macrophages, M2 macrophages, cell cycle found to be substantially downregulated and upregulated, respectively, in HT-29 cells treated with M1 CM, making them unlikely as mediators of reduced 5-FU cytotoxicity. Among cell cycle regulating proteins, p21 was induced in HT-29 cells, but not in CACO-2 cells, in response to M1 CM treatment. However, small interfering RNA (siRNA) knockdown of p21 had no effect on the M1 CM induced cell cycle arrest seen in HT-29 and neither did it change the growth recovery after combined treatment of HT-29 cells with M1 CM and 5-FU. In conclusion, treatment of HT-29 cells with M1 CM reduces the cytotoxic effect of 5-FU and this is mediated by a M1 CM induced cell cycle arrest in the $G_{0} / G_{1}$ and $G_{2} / M$ phases. So far, we lack an explanation why this action is absent in the CACO-2 cells. The current findings may be important for optimization of chemotherapy in colon cancer.

\section{Introduction}

Colorectal cancer (CRC) is one of the most prevalent cancers in the Western world and it is the second cause of cancer deaths in Europe (1). In solid cancers including CRC, immune cells in the tumor stroma play an important role in cancer progression, participating in the regulation of processes such as cancer cell proliferation, immune suppression and invasion/metastasis (2-4). In the stroma of CRC, the tumor-associated macrophages (TAMs) are an important cell type suggested to modulate cancer progression (5-7).

Macrophages can display different phenotypes depending on their microenvironment and are classified as classically activated pro-inflammatory M1 macrophages, or alternatively activated anti-inflammatory M2 macrophages (8-10). The M1 phenotype results from the activation by lipopolysaccharide (LPS) and/or interferon- $\gamma(\mathrm{IFN}-\gamma)$ and release pro-inflammatory factors such as tumor necrosis factor- $\alpha$, interleukin (IL)-6, and IL-12, and cytotoxic substances such as reactive oxygen and nitrogen species (11). The M2 phenotype, on the other hand results from a polarization by cytokines such as IL-4 or IL-13, and release anti-inflammatory cytokines, e.g., IL-10 and transforming growth factor- $\beta$, and angiogenic factors such as vascular endothelial growth factor (12-14). Due to the notion that the macrophages display a broad functional spectrum as well as an ability to change function 
depending on the microenvironment the effect of TAMs on cancer progression is difficult to predict, and results regarding a relationship between TAM levels and prognosis in CRC are contradictory $(6,7,15-18)$. Such conflicts could, at least in part, be explained by how these macrophages have been polarized by the tumor microenvironment (19). Moreover, presence of TAMs in the tumor microenvironment might not only affect tumor progression, but also the response to treatments like e.g., chemotherapy or radiation therapy (10).

Colon cancer is always treated by surgery and, depending on tumor stage, is often supplemented with adjuvant 5-fluorouracil (5-FU) based chemotherapy. Since 5-FU has limited antitumor activity on its own, combinational therapy, such as the FOLFOX treatment is often used, which also includes oxaliplatin and leucovorin, to enhance the antitumor activity of the treatment $(20,21)$.

$5-\mathrm{FU}$ is a chemotherapeutic agent classified as an antimetabolite that primarily acts by the irreversible inhibition of thymidylate synthetase (TS) leading to deoxythymidine monophosphate (dTMP) shortage and non-functional DNA synthesis. Thereby, 5-FU is primarily an S-phase specific drug that acts on actively proliferating cells. Therefore, factors regulating cell proliferation or the cell cycle can modulate 5-FU cytotoxicity (22). Another factor that can affect 5-FU cytotoxicity is the expression levels of various enzymes responsible for intracellular metabolism of 5-FU, which can either result in the primary active substance, fluorodeoxyuridine monophosphate (FdUMP) or the non-active substance, dihydrofluorouracil $(23,24)$. We recently reported that M1 macrophages can reduce proliferation and induce cell cycle arrest of colon cancer cells, as investigated with the HT-29 cell line, in vitro (25). As a follow-up, the aim of the current study was to examine whether conditioned media (CM) from human M1 or M2 macrophages could affect the efficacy of 5-FU treatment of colon cancer cells. Specifically, we investigated effects on proliferation, cell cycle distribution and expression of cell cycle regulating genes and 5-FU metabolic genes in the colon cancer cell lines HT-29 and CACO-2.

\section{Materials and methods}

Cell culture. The colon cancer cell lines, HT-29 and CACO-2, were purchased from DSMZ (Braunschweig, Germany). Each cell line was cultured in RPMI-1640 (RPMI; Life Technologies, Carlsbad, CA, USA) supplemented with $2 \mathrm{mM}$ L-glutamine, $100 \mathrm{U} / \mathrm{ml}$ penicillin and $100 \mu \mathrm{g} / \mathrm{ml}$ streptomycin (Life Technologies) with $10 \%$ heat-inactivated fetal calf serum (FCS) (Thermo Fisher Scientific, Inc., Waltham, MA, USA) and $10 \mathrm{mM}$ HEPES. Both cell lines were grown at $37^{\circ} \mathrm{C}$ in a humidified atmosphere and $5 \% \mathrm{CO}_{2}$.

For all experiments, 29,000 HT-29 cells/well or 19,000 CACO-2 cells/well were seeded onto 24-well plates (Greiner Bio-One GmbH, Frickenhausen, Germany) in $0.5 \mathrm{ml}$ RPMI $10 \%$ FCS plus $10 \mathrm{mM}$ HEPES and cultured for 3 days. Thereafter, cells were treated with M1 or M2 macrophage $\mathrm{CM}$ (for preparation see below) or 5-FU, alone or in combination, according to the schedule shown in Fig. 1.

Isolation of human monocytes and differentiation to macrophages. Buffy coats from healthy blood donors were obtained
Cell count determination

Cell cycle analysis

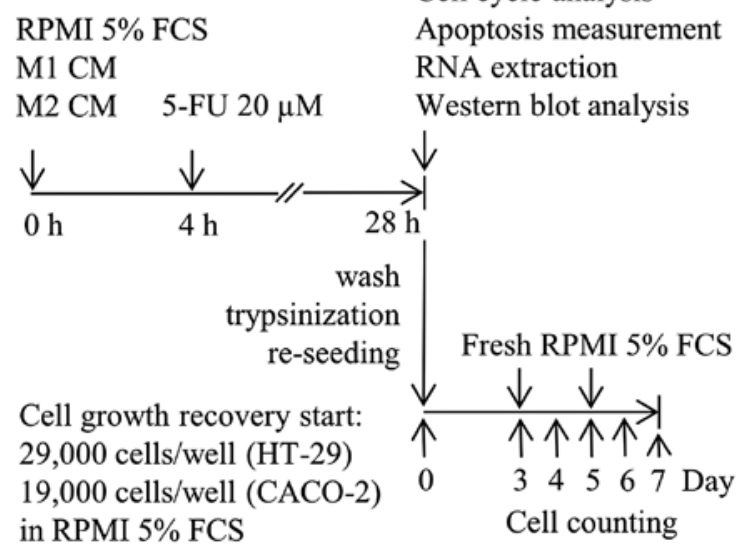

Figure 1. Treatment schedule for experiments performed with HT-29 or CACO-2 cells in the present investigation. Cells were treated as indicated, and in case of combined treatment, 5 -fluorouracil (5-FU) $(20 \mu \mathrm{M})$ was added after $4 \mathrm{~h}$. All experiments were terminated after a total time of $28 \mathrm{~h}$. For cell growth recovery experiments, instead of termination, the cells were washed with RPMI, detached by trypsinization, counted, and re-seeded in RPMI 5\% fetal calf serum (FCS), indicated as day 0 . Each day between day 3 and 7 , duplicate wells of each treatment were terminated for assessment of cell growth recovery.

from the division of Clinical Immunology and Transfusion Medicine, Uppsala University Hospital (Uppsala, Sweden), and monocytes were isolated by gradient centrifugation and allowed to differentiate into macrophages with macrophage colony-stimulating factor (M-CSF) treatment for 6 days, as described previously (25). After macrophage differentiation, the macrophages were further differentiated into M1 macrophages through the addition of $100 \mathrm{ng} / \mathrm{ml}$ LPS (Sigma-Aldrich, St.Louis, MO, USA) plus $20 \mathrm{ng} / \mathrm{ml}$ IFN $-\gamma$ for $48 \mathrm{~h}$ or M 2 macrophages through the addition of $20 \mathrm{ng} / \mathrm{ml} \mathrm{IL-4} \mathrm{plus} 20 \mathrm{ng} / \mathrm{ml}$ IL-13 (all from R\&D Systems, Minneapolis, MN, USA) for $48 \mathrm{~h}$. The differentiated M1 and M2 macrophages [the phenotypes were characterized as described previously (25)] were washed twice with PBS and were cultured for another $48 \mathrm{~h}$ in RPMI 5\% FCS (without either IFN- $\gamma / \mathrm{LPS}$ or IL-4/IL-13) to generate M1 and M2 CM. The collected CM was centrifuged to remove cell debris and stored in aliquots at $-20^{\circ} \mathrm{C}$.

Proliferation studies and cell growth recovery assessment. HT-29 or CACO-2 cells were cultured as described above in the cell culture section and treated as described in Fig. 1 and counted in a hemocytometer. For growth recovery assessment, treated cells were washed, detached by trypsinization, counted in a hemocytometer and subsequently re-seeded at 29,000 HT-29 cells/well or 19,000 CACO-2 cells/well for each treatment onto 24-well cell culture plates (Greiner Bio-One $\mathrm{GmbH}$ ) in $0.5 \mathrm{ml}$ RPMI 5\% FCS. Cells were thereafter counted in a hemocytometer at day 3-7 after treatment. Media renewal was done at day 3 and 5 .

Cell cycle analysis. HT-29 or CACO-2 cells were cultured as described above and were treated as described in Fig. 1. Subsequently, the cells were detached by trypsinization and were pooled with their corresponding culture media possibly containing loose cells. The cells were washed with PBS 
containing $1 \%$ bovine serum albumin (PBS/BSA) and were resuspended in $450 \mu \mathrm{l}$ ice-cold $\mathrm{PBS} / \mathrm{BSA}$ prior to fixation in $5 \mathrm{ml}$ ice-cold $70 \%$ ethanol. Samples were stored at $-20^{\circ} \mathrm{C}$ until analysis.

Before analysis, Triton $\mathrm{X}-100$ was added to a final concentration of $0.1 \%(\mathrm{v} / \mathrm{v})$ and the cells were incubated for $10 \mathrm{~min}$ at $6^{\circ} \mathrm{C}$. Next, the cells were washed twice with $\mathrm{PBS} / \mathrm{BSA}$ and were resuspended in $1 \mathrm{ml}$ PBS/BSA containing $0.1 \%$ Triton $\mathrm{X}-100,50 \mu \mathrm{g} / \mathrm{ml}$ propidium iodide and $200 \mu \mathrm{g} / \mathrm{ml}$ RNase A (both from Sigma-Aldrich). Samples were incubated at room temperature for $45 \mathrm{~min}$ in the dark prior to analysis on a FACSCalibur (BD Biosciences, San Jose, CA, USA) flow cytometer. Cell cycle distributions were calculated using the ModFit LT software v.3.1 (Verity Software House, Inc., Topsham, ME, USA).

RNA extraction and cDNA synthesis. HT-29 and CACO-2 cells were cultured as described above and were treated as described in Fig. 1. Cells were detached by trypsinization and total RNA extracted using the RNeasy Mini kit (Qiagen, Hilden, Germany) according to manufacturer's instructions. RNA quantity and purity, respectively, was determined by measuring the absorbance at $260 \mathrm{~nm}$ and the 260/280 $\mathrm{nm}$ ratio, respectively, in a nanoquant plate analyzed with the infinite M200 Pro plate reader (Tecan, Männedorf, Switzerland). cDNA was transcribed from $1 \mu \mathrm{g}$ total RNA using the High-Capacity cDNA Reverse Transcription kit (Applied Biosystems, Foster City, CA, USA) according to manufacturer's instructions.

Quantitative PCR ( $q P C R)$. qPCR was run on a StepOnePlus Real-Time PCR using Power SYBR-Green Master Mix (both from Applied Biosystems) with a reaction volume of $25 \mu \mathrm{l}$ including $1 \mu \mathrm{l}$ cDNA and $200 \mathrm{nM}$ of each primer (Table I for sequences). All reactions were run in triplicates. Fold change of treated sample vs. untreated control was calculated with REST2009 software (Qiagen) (26) using both GAPDH and POLR2F as housekeeping genes for HT-29 samples and GAPDH, POLR2F, RPL37A and $\beta$-actin for CACO-2 samples. The efficiency of primers was calculated using LinRegPCR software (27). The size of amplified qPCR products was validated by agarose gel-electrophoresis for all primer pairs.

Apoptosis measurement. HT-29 and CACO-2 cells were cultured as described above and were treated as described in Fig. 1. Cells were detached by trypsinization and pooled with their corresponding cell culture media possibly also containing floating cells, were centrifuged at $300 \mathrm{x}$ g for $5 \mathrm{~min}$ and then resuspended in $1 \%$ paraformaldehyde in PBS. Cell suspensions were incubated on ice for $45 \mathrm{~min}$. Next, cells were washed twice with $5 \mathrm{ml}$ PBS and were resuspended in $450 \mu \mathrm{l}$ ice-cold PBS prior to cell fixation in $5 \mathrm{ml}$ ice-cold $70 \%$ ethanol. Fixed cells were stored at $-20^{\circ} \mathrm{C}$ until apoptosis measurements were done using a terminal deoxynucleotidyl transferase dUTP nick end labeling (TUNEL) kit (Phoenix Flow Systems, San Diego, CA, USA) according to manufacturer's instructions. Cell apoptosis was analyzed on a FACSCalibur flow cytometer and acquired data were analyzed with Cell Quest v.3.3 (both from BD Biosciences). p21 siRNA gene knockdown. HT-29 cells were cultured as described above. After 2 days of culture, the culture medium was changed to RPMI 5\% FCS and small interfering RNA (siRNA) against p21 (Silencer Select ID s415) (30 nM) or negative scramble sequence (Silencer Select Negative Control no. 1) (30 nM) were added together with the lipofectamine RNAiMax Transfection Reagent dissolved in Opti-MEM medium according to the manufacturer's instructions (all from Life Technologies). Following a $24 \mathrm{~h}$ transfection, the transfection media were removed and cells were treated as described in Fig. 1 and effects of p21 siRNA knockdown were analysed with respect to cell count, cell growth recovery, cell cycle distribution, and p21 expression.

Immunoblotting. For whole cell lysates, HT-29 and CACO-2 cells were cultured as described above. Samples from siRNA experiments were treated as described in the p21 siRNA knockdown section. For non-siRNA experiments, HT-29 or CACO-2 cells were treated for $28 \mathrm{~h}$ with RPMI 5\% FCS or M1 CM. After treatment, the cells were detached by trypsinization, counted, pelleted, and were resuspended in $1 \mu 1 / 10,000$ cells of Laemmli sample buffer with protease inhibitors [20 $\mu \mathrm{g} / \mathrm{ml}$ aprotinin, $10 \mu \mathrm{g} / \mathrm{ml}$ leupeptin, $5 \mathrm{mM}$ phenylmethylsulfonyl fluoride (all from Sigma-Aldrich)]. Lysed samples were homogenized through a 21 gauge syringe needle, boiled for $5 \mathrm{~min}$ and were stored in aliquots at $-20^{\circ} \mathrm{C}$ until analysis.

Samples $(20 \mu \mathrm{l})$ were subjected to SDS-PAGE $(12 \%$ acrylamide gel) and proteins were transferred onto a PVDF membrane (Bio-Rad, Hercules, CA, USA). Blocking was performed for $1 \mathrm{~h}$ at room temperature with 5\% BSA in TBS $0.1 \%$ Tween-20 (TBS-T). Membranes were incubated overnight at $4^{\circ} \mathrm{C}$ with p21 antibodies (12D1 rabbit monoclonal antibody; Cell Signaling Technology, Inc, Danvers, MA, USA) diluted 1:1,000 in blocking solution. The secondary antibody (goat anti-rabbit IgG-HRP, Sc-2004; Santa Cruz Biotechnology, Inc., Dallas, TX, USA) was diluted 1:15,000 in TBS-T and was applied for $1 \mathrm{~h}$ at room temperature. Signals were developed using SuperSignal West Pico Chemiluminescent Substrate (Thermo Fisher Scientific, Inc.) and light intensities were detected by exposure to Amersham Hyperfilm ECL (GE Healthcare, Little Chalfont, UK). After detection of p21, membranes were stripped using Restore Western Blot Stripping Buffer (Thermo Fisher Scientific, Inc.) and were incubated with $\beta$-actin antibody as loading control $(\mathrm{N}-21$ rabbit polyclonal antibody; Santa Cruz Biotechnology, Inc.) diluted 1:500 in blocking solution.

Statistics. All the results are presented as mean value \pm standard deviation (SD). Two-sided Student's t-test was used for the statistical analyses. All experiments with macrophage $\mathrm{CM}$ were performed with at least three different macrophage batches generated from different donors.

\section{Results}

CM from macrophages of $M 1$ phenotype attenuates 5-FU mediated growth inhibition of HT-29 cells, but not of CACO-2 cells. Dose-response analysis of a $24 \mathrm{~h}$ 5-FU treatment of HT-29 or CACO-2 cells revealed similar inhibition of growth 
Table I. Primer sequences used for qPCR.

\begin{tabular}{|c|c|c|c|}
\hline $\begin{array}{l}\text { Gene } \\
\text { name }\end{array}$ & $\begin{array}{l}\text { Forward primer } \\
\text { sequence } 5^{\prime} \rightarrow 3^{\prime}\end{array}$ & $\begin{array}{l}\text { Reverse primer } \\
\text { sequence } 5^{\prime} \rightarrow 3^{\prime}\end{array}$ & $\begin{array}{c}\text { Genebank } \\
\text { accession no. }\end{array}$ \\
\hline$\beta$-actin & ATTGCCGACAGGATGCAGAA & GCTGATCCACATCTGCTGGAA & NM_001101.3 \\
\hline CCNB1 & AACTTTCGCCTGAGCCTATTTT & TTGGTCTGACTGCTTGCTCTT & NM_031966.3 \\
\hline CCND1 & CAATGACCCCGCACGATTTC & CATGGAGGGCGGATTGGAA & NM_053056.2 \\
\hline CCNE1 & GCCAGCCTTGGGACAATAATG & CTTGCACGTTGAGTTTGGGT & NM_001238.2 \\
\hline CCNG2 & TCTGTATTAGCCTTGTGCCTTCT & CCTTGAAACGATCCAAACCAAC & NM_004354.2 \\
\hline CDC25A & GTGAAGGCGCTATTTGGCG & TGGTTGCTCATAATCACTGCC & NM_001789.2 \\
\hline $\mathrm{CDC} 25 \mathrm{C}$ & ATGACAATGGAAACTTGGTGGAC & GGAGCGATATAGGCCACTTCTG & NM_001790.3 \\
\hline CDK1 & AAACTACAGGTCAAGTGGTAGCC & TCCTGCATAAGCACATCCTGA & NM_001786.4 \\
\hline $\mathrm{CDK} 2$ & GTACCTCCCCTGGATGAAGAT & CGAAATCCGCTTGTTAGGGTC & NM_001798.3 \\
\hline CDK4 & CTGGTGTTTGAGCATGTAGACC & GATCCTTGATCGTTTCGGCTG & NM_000075.3 \\
\hline CDK6 & CCAGATGGCTCTAACCTCAGT & AACTTCCACGAAAAAGAGGCTT & NM_001145306.1 \\
\hline CDKN3 & TCCGGGGCAATACAGACCAT & GCAGCTAATTTGTCCCGAAACTC & NM_005192.3 \\
\hline CHK1 & ATATGAAGCGTGCCGTAGACT & TGCCTATGTCTGGCTCTATTCTG & AF016582.1 \\
\hline CHK2 & TTATCTGCCTTAGTGGGTATCCA & CTGTCGTAAAACGTGCCTTTG & NM_001005735.1 \\
\hline DPD & GGCGGACATCGAGAGTATCCT & TTCTTGGCCGAAGTGGAACAC & NM_000110.3 \\
\hline FOXO1 & GGATGTGCATTCTATGGTGTACC & TTTCGGGATTGCTTATCTCAGAC & NM_002015.3 \\
\hline FOXO3 & CGGACAAACGGCTCACTCT & GGACCCGCATGAATCGACTAT & NM_001455.3 \\
\hline GADD45A & GAGAGCAGAAGACCGAAAGGA & CAGTGATCGTGCGCTGACT & NM_001924.3 \\
\hline GAPDH & CAACAGCGACACCCACTCCT & CACCCTGTTGCTGTAGCCAAA & NM_002046.4 \\
\hline MTHFR & GAGCGGCATGAGAGACTCC & CCGGTCAAACCTTGAGATGAG & NM_005957.4 \\
\hline p21 & TTAGCAGCGGAACAAGGAGT & AGCCGAGAGAAAACAGTCCA & NM_000389.4 \\
\hline p27 & TAATTGGGGCTCCGGCTAACT & TGCAGGTCGCTTCCTTATTCC & NM_004064.3 \\
\hline p57 & GCGGCGATCAAGAAGCTGT & GCTTGGCGAAGAAATCGGAGA & NM_001122631.1 \\
\hline p16 & ATGGAGCCTTCGGCTGACT & GTAACTATTCGGTGCGTTGGG & NM_000077.4 \\
\hline POLR2F & ATGTCAGACAACGAGGACAATTT & TTCGGCATTCTCCAAGTCATC & NM_021974.3 \\
\hline RPL37A & ATTGAAATCAGCCAGCACGC & AGGAACCACAGTGCCAGATCC & NM_000998.4 \\
\hline TP & GGTGTGGGTGACAAGGTCAG & GCAGCACTTGCATCTGCTC & NM_001953.4 \\
\hline TP53 & CAGCACATGACGGAGGTTGT & TCATCCAAATACTCCACACGC & NM_000546.5 \\
\hline TS & CTGCTGACAACCAAACGTGTG & GCATCCCAGATTTTCACTCCCTT & NM_001071.2 \\
\hline UMPS & GTGTGTGGAGTGCCTTATACAG & CСТTCTACAAGACGCTTAGTTCC & NM_000373.3 \\
\hline WEE1 & GACGAAGATGATTGGGCATCC & TGGACTGGAGATCCTTGTTACA & NM_001143976.1 \\
\hline
\end{tabular}

qPCR, quantitative PCR; CCN, cyclin; CDC, cell division cycle; $C D K$, cyclin-dependent kinase; CDKN, cyclin-dependent kinase inhibitor; CHK, checkpoint division cycle; DPD, dihydropyrimidine dehydrogenase; FOXO, forkhead box O; GADD, growth arrest and DNA-damage-inducible; GAPDH, glyceraldehyde-3-phosphate dehydrogenase; MTHFR, methylenetetrahydrofolate reductase; POLR2F, polymerase (RNA) II (DNA directed) polypeptide F; RPL37A, ribosomal protein L37a; TP, thymidine phosphorylase; TP53, tumor protein p53; TS, thymidylate synthetase; UMPS, uridine monophosphate synthetase.

for either cell line with a partial inhibition at $1 \mu \mathrm{M} 5-\mathrm{FU}$ (cell count reduced to about $80 \%$ of control) and reaching a plateau at about $10 \mu \mathrm{M} 5$-FU (cell count reduced to about $60 \%$ of control) (Fig. 2A). For all further experiments $20 \mu \mathrm{M} \mathrm{5-FU}$ was chosen for treatment of either cell line.

In accordance with previously published results (25), M1 CM induced a strong reduction in growth of both HT-29 and CACO-2 cells when treated for $28 \mathrm{~h}$ whereas treatment with M2 CM had no significant effect (Fig. 2B and C). No apparent additive effect in reduction of cell growth could be seen when cells were treated with both 5-FU and M1 CM (4 h with $\mathrm{M} 1 \mathrm{CM}+24 \mathrm{~h}$ in combination with 5-FU). To further evaluate the effect of these treatments on cell growth, we re-seeded the treated cells in RPMI 5\% FCS and studied their growth for 7 days. Both HT-29 and CACO-2 cells having been treated with 5-FU alone showed poor recovery of cell growth (Fig. 2D and E). HT-29 cells having been treated with M1 or M2 CM showed similar growth recovery as the untreated control while CACO-2 cells treated with M1 CM had a marked reduction in cell growth recovery, with cell count reduced to about $30 \%$ of a control after 7 days of recovery (Fig. 2D and E). Interestingly, HT-29 cells having been treated with 5-FU combined with M1 CM (4 h with $\mathrm{M} 1 \mathrm{CM}+24 \mathrm{~h}$ combined with 5-FU) recovered their growth to a much greater extent than HT-29 cells treated with 5-FU alone or 5-FU in combination with M2 CM (Fig. 2D). In 
A

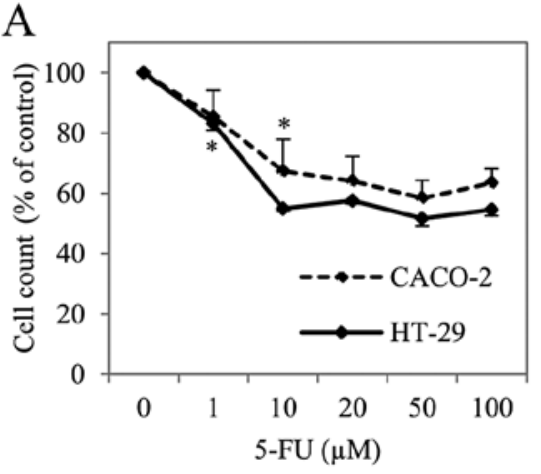

$\mathrm{B}$

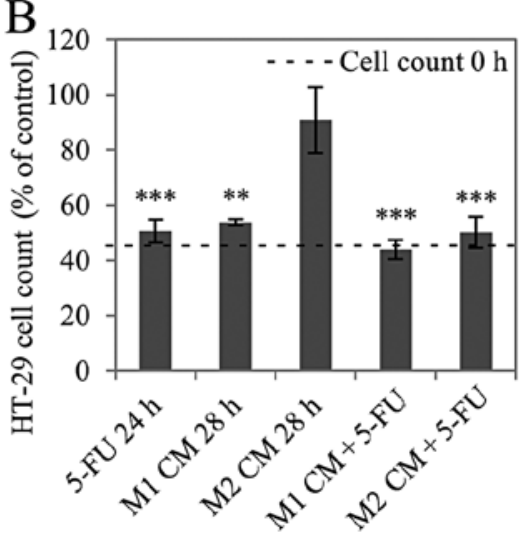

$\mathrm{C}$

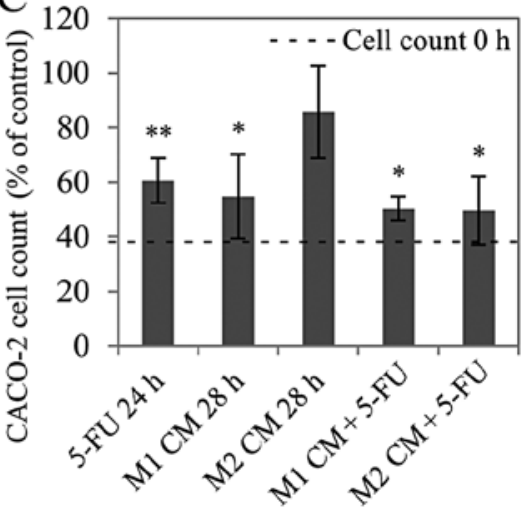

\section{$\mathrm{D}$}

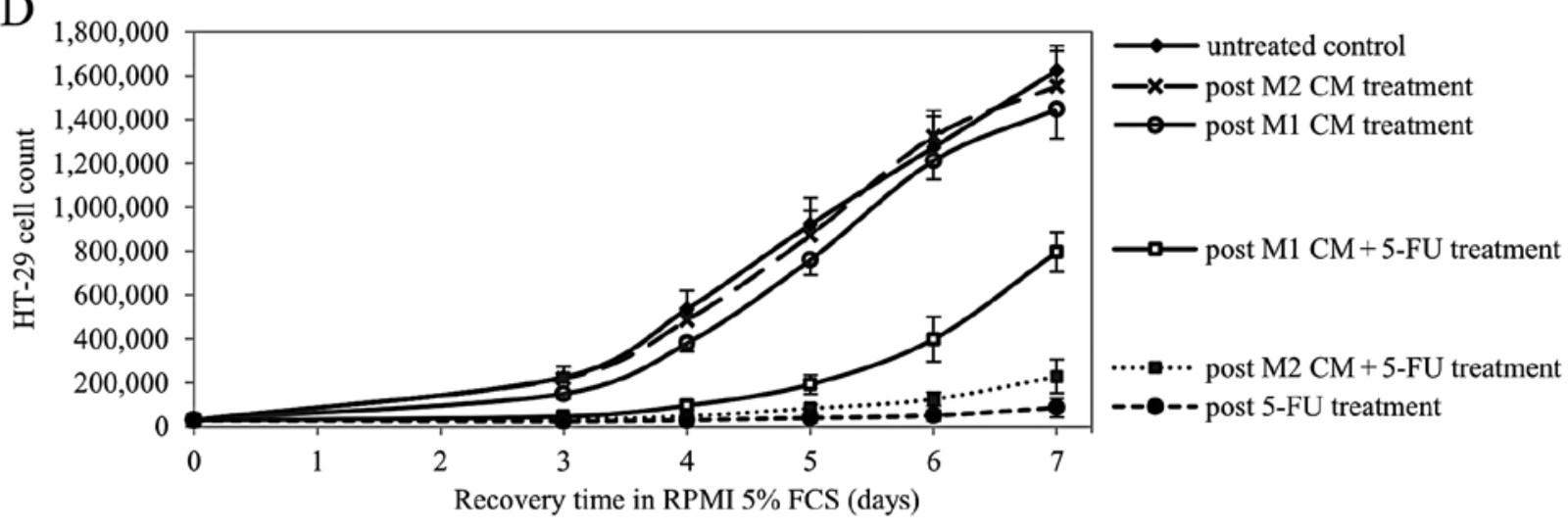

$\mathrm{E}$

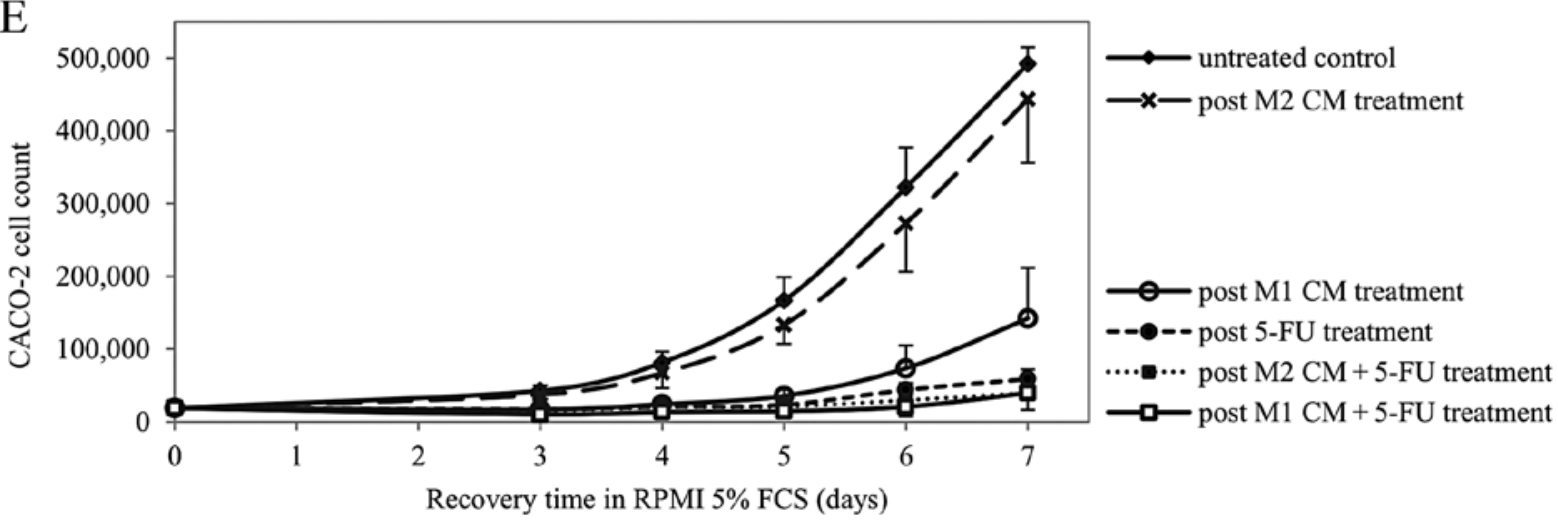

Figure 2. Effect of 5-fluorouracil (5-FU) and conditioned media (CM) from macrophages of M1 or M2 phenotype on the proliferation of (A and B) HT-29 cells and (A and C) CACO-2 cells. Cell growth recovery after indicated treatments of (D) HT-29 cells and (E) CACO-2 cells. Cells were treated as described in Fig. 1 (Materials and methods section). The growth recovery experiments were conducted on treated cells detached by trypsinization and were re-seeded (HT-29: 29,000 cells/well, CACO-2: 19,000 cells/well) in RPMI 5\% fetal calf serum (FCS) and counted day 3-7 after re-seeding. Results are shown as mean value \pm standard deviation (SD). (A) $n \geq 3$ independent experiments. (B and D) At least four independent experiments with four different macrophage batches from different donors were used. (C and E) At least three independent experiments with three different macrophage batches from different donors were used. The dotted line in (B and C) indicate cell number at the start of treatment. ${ }^{*} \mathrm{P}<0.05,{ }^{* *} \mathrm{p}<0.01,{ }^{* * *} \mathrm{p}<0.001$.

contrast, CACO-2 cells having been treated with a combination of 5-FU and M1 CM showed poor growth recovery, similar to that of 5-FU treatment alone (Fig. 2E). There was no induction of apoptosis observed in either HT-29 or CACO-2 cells treated with 5-FU (20 $\mu \mathrm{M}, 24 \mathrm{~h}), \mathrm{M} 1$ or M2 CM (28 h) as determined by TUNEL assay (results not shown, $n=3-6$ independent experiments).

CM from macrophages of $M 1$ phenotype reduces the 5-FU dependent accumulation of cells in S-phase in HT-29 cells, but not in $\mathrm{CACO}-2$ cells. The cell cycle distribution was analyzed for HT-29 and CACO-2 cells treated with 5-FU, when administered either alone or in combination with either M1 or M2 CM. 5-FU treatment alone, caused accumulation of cells in S-phase and a large reduction of cells in $\mathrm{G}_{2} / \mathrm{M}$ phases in both HT-29 and CACO-2 cells (Table II). In HT-29 cells, treatment with M1 CM caused a substantial decrease of cells in S-phase with an accumulation of cells in $\mathrm{G}_{2} / \mathrm{M}$ and $G_{0} / G_{1}$, while treatment with $M 2 C M$ had no significant effect on the cell cycle distribution, when compared to control 
Table II. Cell cycle analysis of HT-29 cells and CACO-2 cells.

\begin{tabular}{lcccc}
\hline Treatment & Cell line & $\mathrm{G}_{0} / \mathrm{G}_{1}(\%)$ & S-phase $(\%)$ & $\mathrm{G}_{2} / \mathrm{M}(\%)$ \\
\hline RPMI 5\% FCS & HT-29 & $74.1 \pm 5.6$ & $17.7 \pm 2.9$ & $8.2 \pm 3.2$ \\
M1 CM & HT-29 & $82.1 \pm 2.2$ & $2.7 \pm 0.6^{\mathrm{c}}$ & $15.2 \pm 1.8^{\mathrm{c}}$ \\
M1 CM + 5-FU & HT-29 & $64.9 \pm 7.8$ & $26.5 \pm 7.8^{\mathrm{d}}$ & $8.5 \pm 1.8^{\mathrm{d}}$ \\
M2 CM & HT-29 & $80.2 \pm 2.8$ & $13.7 \pm 2.0$ & $6.1 \pm 1.8$ \\
M2 CM + 5-FU & HT-29 & $64.8 \pm 7.8$ & $33.3 \pm 5.5^{\mathrm{b}}$ & $1.9 \pm 2.5^{\mathrm{b}}$ \\
5-FU & HT-29 & $60.5 \pm 5.9^{\mathrm{a}}$ & $38.8 \pm 6.5^{\mathrm{c}}$ & $0.7 \pm 0.9^{\mathrm{b}}$ \\
RPMI 5\% FCS & CACO-2 & $44.8 \pm 2.4$ & $40.4 \pm 0.7$ & $14.8 \pm 2.4$ \\
M1 CM & CACO-2 & $49.8 \pm 4.3^{\mathrm{y}}$ & $36.0 \pm 4.4$ & $14.2 \pm 0.6$ \\
M1 CM + 5-FU & CACO-2 & $45.3 \pm 0.8^{\mathrm{d}}$ & $53.2 \pm 3.3^{\mathrm{b}}$ & $1.5 \pm 2.5^{\mathrm{c}}$ \\
M2 CM & CACO-2 & $43.1 \pm 1.7$ & $43.2 \pm 2.6$ & $13.6 \pm 2.4$ \\
M2 CM + 5-FU & CACO-2 & $49.1 \pm 1.4^{\mathrm{a}}$ & $50.9 \pm 1.4^{\mathrm{c}}$ & $0.0 \pm 0.0^{\mathrm{c}}$ \\
5-FU & CACO-2 & $49.6 \pm 0.3^{\mathrm{b}}$ & $50.4 \pm 0.3^{\mathrm{c}}$ & $0.0 \pm 0.0^{\mathrm{c}}$ \\
\hline
\end{tabular}

Cells were treated with either M1 or M2 CM for $28 \mathrm{~h}, 5-\mathrm{FU}(20 \mu \mathrm{M})$ for $24 \mathrm{~h}$, or combined treatment with $\mathrm{M} 1$ or M2 CM and 5-FU (4 h with $\mathrm{M} 1$ or $\mathrm{M} 2 \mathrm{CM}+24 \mathrm{~h}$ in combination with 5-FU). Results are shown as mean value \pm standard deviation $(\mathrm{SD})(\mathrm{HT}-29: \mathrm{n} \geq 4$ independent experiments, CACO-2: $\mathrm{n} \geq 3$ independent experiments). At least four (three for CACO-2) different macrophage batches from different donors were used. Unpaired two-sided t-tests ${ }^{a} \mathrm{P}<0.05,{ }^{b} \mathrm{p}<0.01,{ }^{\mathrm{c}} \mathrm{p}<0.001$ compare treatments vs. untreated control (RPMI 5\% FCS). ${ }^{\mathrm{d} I n d i c a t e}$ statistical significant difference for $\mathrm{M} 1 \mathrm{CM}+5-\mathrm{FU}$ treatment vs. 5-FU treatment using the unpaired two-sided t-test $(\mathrm{p}<0.05)$. FCS, fetal calf serum; CM, conditioned media; 5-FU, 5-fluorouracil.

cells (Table II). Combined treatment of HT-29 cells with 5-FU and M1 CM revealed a significantly reduced 5-FU induced accumulation of cells in S-phase and retention of cells in $\mathrm{G}_{2} / \mathrm{M}$ when compared to 5-FU alone, while combined treatment with 5-FU and M2 CM revealed cell cycle distributions similar to that of 5-FU alone (Table II). In contrast, for CACO-2 cells, treatment with M1 or M2 CM did not cause any change in the cell cycle distribution when compared to control cells. Furthermore, the combined treatment of CACO-2 cells with 5-FU and M1 or M2 CM showed a cell cycle distribution similar to that of 5-FU alone, indicating that M1 and M2 CM does not affect the cell cycle to any major extent in $\mathrm{CACO}-2$ cells (Table II).

CM from macrophages changes the mRNA expression of some 5-FU metabolic genes in HT-29, but is not consistent with reduced cytostatic effect of 5-FU. The finding of a reduction in efficacy of 5-FU in HT-29 cells in response to a combined treatment with M1 CM could depend on changes in the cell cycle regulation and/or regulation of the metabolism of 5-FU in these cells. We analyzed the mRNA expression of some key enzymes involved in 5-FU metabolism. In HT-29 cells treated with M1 CM, thymidine phosphorylase (TP) was highly upregulated (32-fold increase), TS was substantially downregulated (16-fold decrease) and dihydropyrimidine dehydrogenase (DPD) was slightly downregulated while uridine monophosphate synthetase (UMPS) and methylenetetrahydrofolate reductase (MTHFR) were unaffected as noted in three independent experiments (Fig. 3). The same expression pattern was seen in HT-29 cells treated with
M1 CM in combination with 5-FU. Treatment of HT-29 cells with M2 CM, 5-FU or 5-FU in combination with M2 CM did not change the mRNA expression to any major extent when compared to control. Since the mRNA expression of TS and TP were strongly affected in M1 CM treated HT-29 cells we also examined the mRNA expression of TS and TP in M1 CM treated CACO-2 cells. The same expression pattern as seen in HT-29 cells, although to a lower extent, was also seen in M1 CM treated CACO-2 cells; upregulation of TP (4-fold increase) and downregulation of TS (2-fold decrease) (results not shown).

$C M$ from macrophages of $M 1$ phenotype affects the $m R N A$ expression of cell cycle regulatory genes in HT-29 and CACO-2 cells. The mRNA expression of cell cycle regulatory genes were analyzed in both HT-29 cells and CACO-2 cells after treatment with $\mathrm{M} 1 \mathrm{CM}$ and the results were compared to an untreated control from either cell line. In HT-29 cells, genes that were upregulated $>2$-fold when treated with M1 CM were cyclin (CCN)-D1, cyclin-dependent kinase (CDK)-6, forkhead box O (FOXO)-3, growth arrest and DNA-damage-inducible (GADD)-45A and p21. In contrast, CCNE1, CDK2, checkpoint division cycle (CHK)-1 and CHK2 were downregulated 2-fold or more (Fig. 4A). mRNA expression of some of the genes affected by M1 CM in HT-29 cells (CCNE1, CDK2, p21, FOXO3 and GADD45A) were also analyzed in HT-29 cells after treatment with M2 CM, but none of these showed changes in expression when compared to control cells (results not shown). In CACO-2 cells, we observed some differences in mRNA expression of some cell cycle regulatory proteins in response to M1 CM treatment when compared to that seen in HT-29 cells. p21 was not upregulated in CACO-2 cells treated with M1 CM, and there were no downregulation of CCNE1 and CDK2 (Fig. 4B). There was a slight upregulation of cell division cycle (CDC)-25C and WEE1 in CACO-2 cells which was not seen in HT-29 cells, and also a 2-fold or more upregulation in CCND1, CDK6, p27, FOXO1, FOXO3 and GADD45A, being similar to that seen in HT-29 cells. p16 was undetected in both HT-29 and CACO-2 cells, for both untreated and M1 CM treated cells (results not shown). We also examined the mRNA expression of mutated p53 in HT-29 since it has been suggested to mediate resistance to anticancer drugs (28), however, the p53 expression was not affected by M1 CM treatment (Fig. 4A).

Induced p21 protein expression in HT-29 cells in response to treatment with CM from macrophages of M1 phenotype does not affect the cell cycle or 5-FU cytotoxicity in HT-29 cells. In agreement with the observed mRNA data for p21 (Fig. 4), the expression of $\mathrm{p} 21$ protein was induced by M1 CM treatment of HT-29 cells, but not of CACO-2 cells (Fig. 5). To investigate whether the induction of p21 in HT-29 cells was important for cell cycle arrest and 5-FU resistance, we knocked down the p21 expression with siRNA (Fig. 5). Knockdown of p21 did not reveal any changes in cell cycle distribution, cell proliferation or cell growth recovery in response to treatment with M1 CM and 5-FU, in combination or alone, when compared to negative siRNA scramble sequence control (results not shown, $\mathrm{n}=2$ independent experiments). 


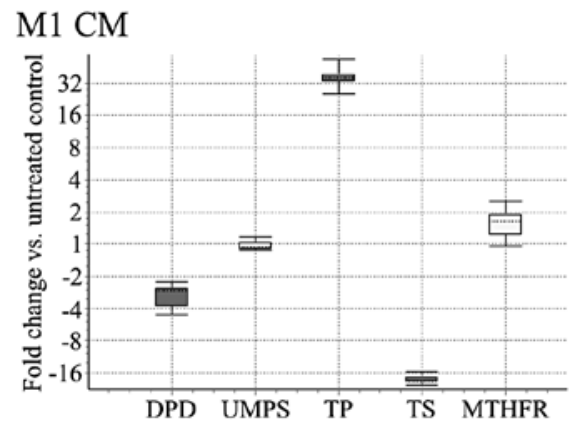

$\mathrm{M} 1 \mathrm{CM}+5-\mathrm{FU}$

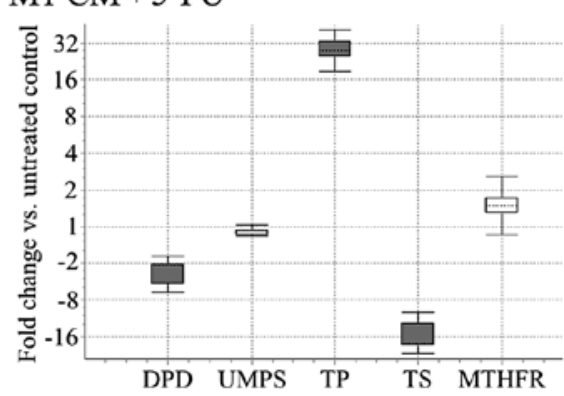

$\mathrm{M} 2 \mathrm{CM}+5-\mathrm{FU}$
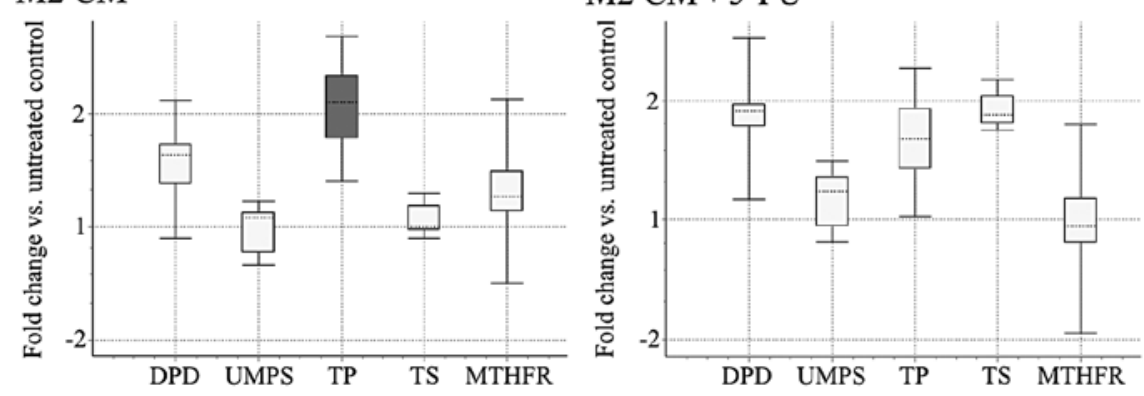

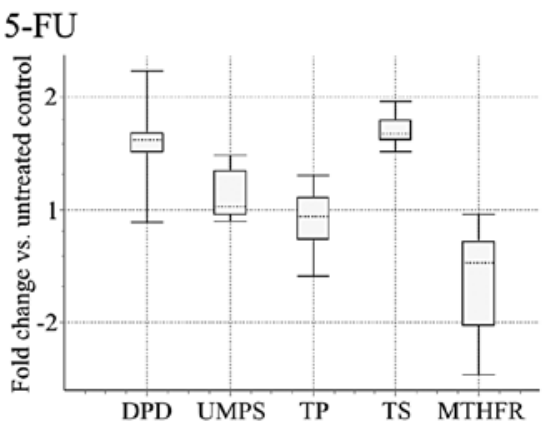

Figure 3. mRNA expression of genes involved in 5-fluorouracil (5-FU) metabolism in HT-29 cells. Cells were treated as indicated with either M1 or M2 conditioned media (CM) for $28 \mathrm{~h}, 5-\mathrm{FU}(20 \mu \mathrm{M})$ for $24 \mathrm{~h}$, or combined treatment with M1 or M2 CM and 5-FU (4 h with M1 or M2 CM + $24 \mathrm{~h}$ in combination with 5-FU). Results are expressed as fold change of treated cells when compared to control cells and presented in whisker box plots. For each gene analyzed, three independent experiments were performed with three different macrophage batches from different donors. Observe different range of the y-axis between treatments. Gray boxes indicate an up- or downregulation of the mean value, 2-fold or more and white boxes indicate an up- or downregulation of the mean value of $<2$-fold.

\section{Discussion}

In this study we have shown that CM from human macrophages of M1 phenotype attenuated the cytotoxic effect of 5-FU in the colon cancer cell line HT-29, but not in the colon cancer cell line CACO-2, and not by CM from the M2 phenotype of macrophages. Treatment of HT-29 cells with 5-FU in combination with $\mathrm{M} 1 \mathrm{CM}$ resulted in growth arrest of either cell line, as expected, because both M1 CM and 5-FU induced growth arrest in HT-29 cells on their own. Interestingly, after removal of $\mathrm{M} 1 \mathrm{CM}$ and 5-FU and continued culture, the HT-29 cells recovered their proliferation to a much larger extent than HT-29 cells having been treated with 5-FU alone or the combined treatment with $\mathrm{M} 2 \mathrm{CM}$ and 5-FU. Although CACO-2 cells exhibited similar growth arrest in response to $\mathrm{M} 1 \mathrm{CM}$ or 5-FU treatment, they did not recover their cell growth after the combined treatment with M1 CM and 5-FU (compare Fig. 2D and E).

5-FU primarily targets proliferating cells, and more specifically cells going through S-phase by depleting dTMP nucleotides thereby leading to failed DNA synthesis (29). Studies have shown that some colon cancer cells can be protected from cytotoxic effects of 5-FU through induction of cell cycle arrest in $\mathrm{G}_{0} / \mathrm{G}_{1}$ and/or $\mathrm{G}_{2} / \mathrm{M}(22,30)$. This seems to be the case for the HT-29 cell line. Thus, HT-29 cells treated with $\mathrm{M} 1 \mathrm{CM}$ were arrested in both $\mathrm{G}_{0} / \mathrm{G}_{1}$ and $\mathrm{G}_{2} / \mathrm{M}$. Treatment of HT-29 cells with 5-FU induced accumulation of cells in S-phase and this accumulation was significantly reduced in HT-29 cells in response to combined treatment with M1 CM and 5-FU. The cell cycle arrest induced by M1 CM treatment is most likely a strong contributing factor for the attenuated cytotoxic effect of 5-FU seen on HT-29 cells. In support of this conclusion, M1 CM treatment of CACO-2 cells did not induce cell cycle arrest, neither did M1 CM treatment protect CACO-2 cells from the cytotoxic effect of 5-FU.

In an attempt to understand the mechanisms behind the cell cycle regulation, we examined how M1 CM treatment of HT-29 and CACO-2 cells affected the mRNA expression of some genes known to participate in cell cycle regulation. Of the genes analyzed, the mRNA expression of p21 was found to be substantially upregulated in M1 CM treated HT-29 cells while seemingly unchanged in CACO-2 cells. Moreover, the increase in p21 was seen also at the protein level in HT-29 cells, but not in CACO-2 cells. p21 function as an inhibitor of multiple CDKs with the ability to induce cell cycle arrest in both $\mathrm{G}_{0} / \mathrm{G}_{1}$ and $\mathrm{G}_{2} / \mathrm{M}(31,32)$ and an increase in $\mathrm{p} 21$ expression has been reported to contribute to 5-FU resistance in cancer cell lines of colon and other origins $(33,34)$. Besides p21 upregulation, M1 CM treatment also induced downregulation of CCNE1 and CDK2 in HT-29, but not in CACO-2. The CCNE1/CDK2 complex is required for G1-S transition, and the complex is blocked by $\mathrm{p} 21$ (35).

The upregulation of $\mathrm{p} 21$ is presumably mediated via a p53-independent pathway since HT-29 cells has a mutated TP53 gene (R273H mutation) (36). Although the mutated p53 has lost its ability to induce p21 expression, it has been shown to gain functions affecting cell proliferation and resistance to anticancer drugs (28). We therefore examined the mutated p53 mRNA expression in HT-29 cells treated with M1 CM, but no change in expression was observed when compared to control. The FOXO transcription factors can regulate p21 gene expression independently of p53 (37). Both FOXO1 and FOXO3 were upregulated in HT-29 cells when treated with M1 CM and could be factors that induce p21 expression in HT-29 
A
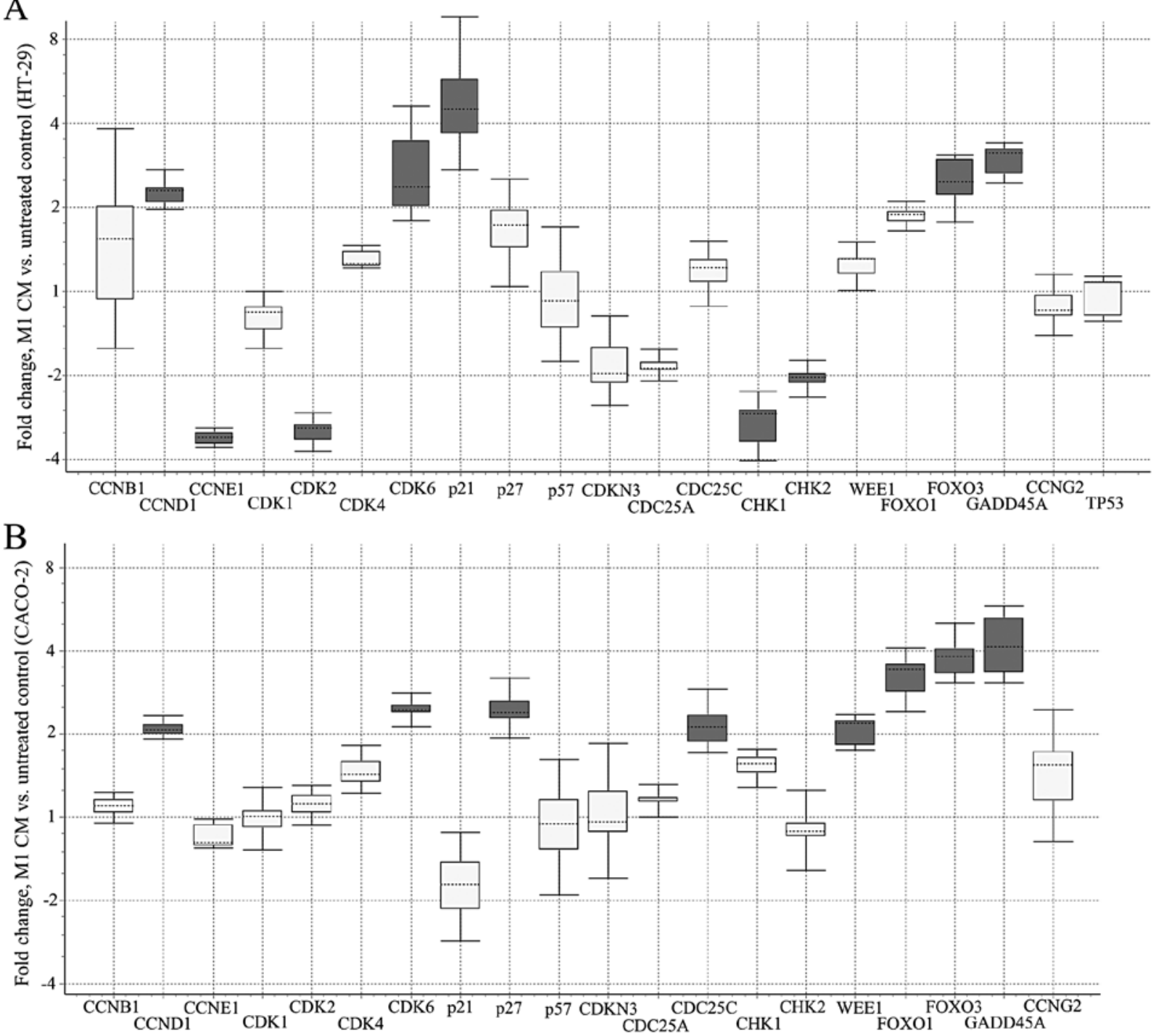

Figure 4. mRNA expression of genes involved in cell cycle regulation in (A) HT-29 cells and (B) CACO-2 cells treated with M1 conditioned media (CM) for $28 \mathrm{~h}$. Results are expressed as fold change in response to M1 CM treatment compared to control and presented in whisker box plots. For each gene analyzed, three independent experiments were performed using three different macrophage batches from different donors. Gray boxes indicate a mean up- or downregulation 2-fold or more and white boxes indicate a mean up- or downregulation <2-fold. CCN, cyclin; CDC, cell division cycle; CDK, cyclin-dependent kinase; CDKN: cyclin-dependent kinase inhibitor, $\mathrm{CHK}$, checkpoint division cycle; FOXO: forkhead box O, GADD: growth arrest and DNA-damage-inducible.

A

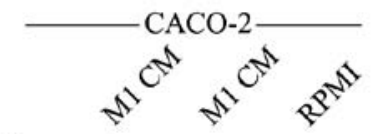

M1 CM batch

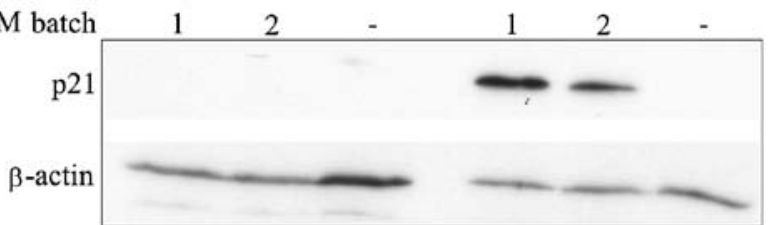

B

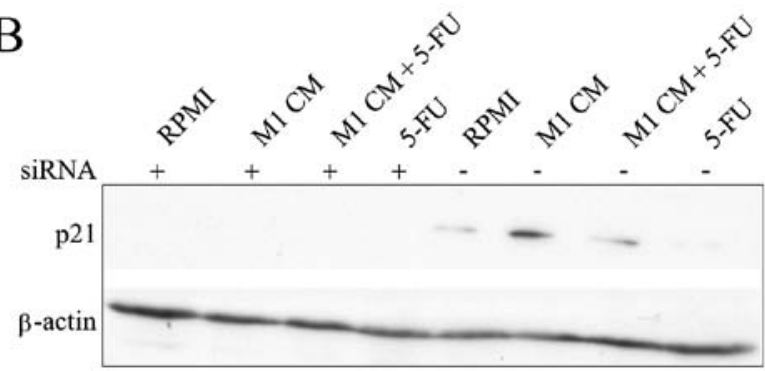

Figure 5. Western blotting of p21. (A) CACO-2 and HT-29 cells treated with M1 conditioned media (CM) for $28 \mathrm{~h}$ or untreated controls (RPMI) and subjected to western blotting. Treatment with two different batches of M1 CM are shown in (A) and the results are representative of four different batches of M1 CM. (B) HT-29 cells treated with $30 \mathrm{nM}$ small interfering RNA (siRNA) against p21 (siRNA+) or negative scramble sequence (siRNA-) and further treated with M1 CM for $28 \mathrm{~h}$, 5-fluorouracil (5-FU) $(20 \mu \mathrm{M})$ for $24 \mathrm{~h}$ or M1 CM +5 -FU (4 h with M1 CM $+24 \mathrm{~h}$ in combination with 5-FU) and subjected to western blotting. The results in (B) are from one representative experiment out of two. $\beta$-actin was used as loading control.

cells. However, FOXO1 and FOXO3 were upregulated also in CACO-2 cells, but without any increase in p21 expression. FOXO transcription factors have also been reported to induce transcription of the cell cycle inhibitors, p27, GADD45A and CCNG2 (38-40). GADD45A and p27 were upregulated in both HT-29 and CACO-2 cells but since CACO-2 cells did not show 
cell cycle arrest, GADD45A and p27 appear to be less likely mediators of cell cycle arrest in HT-29 cells.

Using p21 knockdown experiments, we investigated whether p21 could be a major contributor to the cell cycle arrest seen in HT-29 cells treated with M1 CM. The M1 CM induced p21 protein expression in HT-29 cells was inhibited by p21 siRNA treatment (Fig. 5). However, the knockdown of p21 did not affect the cell cycle arrest induced by M1 CM, nor did it affect the proliferation of HT-29 cells or the growth recovery after M1 CM, 5-FU or combined M1 CM and 5-FU treatment. These results, therefore, strongly suggest that p21 is not a major contributor to the cell cycle arrest induced by M1 CM.

Since 5-FU needs to be metabolized inside the cell in order to become cytotoxic, we reasoned that M1 CM might also affect the expression of enzymes involved in 5-FU metabolism in a manner that could contribute to an attenuated effect of 5-FU on HT-29 cells.

A series of enzymes are involved in the conversion of 5-FU into its primary active component FdUMP which binds to the nucleotide-binding site of TS and thereby causes the inactivation of this enzyme (24). TS andFdUMP form a complex together with 5,10-methylenetetrahydrofolate (MTHF) and a high concentration of MTHF improves the response of 5-FU, which is the reason why the MTHF precursor, leucovorin, often is used in combination with 5-FU (41). MTHFR is a key enzyme in the folate metabolism that converts MTHF into 5-methyltetrahydrofolate, therefore, high levels of MTHFR could reduce the effect of 5-FU. TP converts 5-FU into 5-fluorodeoxyuridine which is further converted into FdUMP (42). 5-FU can also be converted into fluorouridine triphosphate(FUTP) which contributes to5-FU toxicity through its incorporation into RNA (43). FUTP is created through phosphorylation of 5-FU to fluorouracil monophosphate, primarily via UMPS which is further phosphorylated to FUTP (44). The rate-limiting catabolic enzyme for 5-FU is DPD that converts 5-FU into dihydrofluorouracil, and high DPD activity can increase resistance to 5-FU (45).

Of the key enzymes in 5-FU metabolism analyzed in HT-29 cells, several were regulated at the mRNA level by M1 CM, but not in a manner that would imply an increase in resistance to 5-FU. Thus, e.g., DPD was downregulated by M1 CM. Furthermore, TP was highly upregulated (32-fold increase) by M1 CM and TS was highly downregulated (16-fold decrease). Both upregulation of TP and downregulation of TS has been reported to increase the cytotoxic effect of 5-FU $(46,47)$, which is the opposite of what we see in M1 CM treated HT-29 cells. In addition, CACO- 2 cells, which did not change their responsiveness to 5-FU following M1 CM treatment also showed downregulation of TS and upregulation of TP mRNA. When taken together, the upregulation of TP and the downregulations of TS and DPD all seem unlikely to be responsible for the reduced responsiveness to 5-FU seen in HT-29 cells treated with M1 CM.

In conclusion, our results show that treatment of HT-29 cells with M1 CM attenuates the cytotoxic action of 5-FU and that this effect is mediated by M1 CM-induced cell cycle arrest in $G_{0} / G_{1}$ and $G_{2} / M$. We cannot, so far, offer a molecular mechanism of action of this attenuating effect of M1 CM on 5-FU cytotoxicity. Interestingly, this effect could not be mimicked in another colon cancer cell line, CACO-2, albeit the fact that this cell line responded similarly to HT-29 on a single treatment with M1 CM or 5-FU. This lack of mimicry remains to be elucidated. The current findings may imply that the efficacy of 5-FU based chemotherapy for some colon cancer patients could be, in part, dependent on the phenotype of the macrophages residing in the tumor stroma. Further studies along the current line of investigations are warranted in order to optimize chemotherapy in e.g., colon cancer.

\section{Acknowledgements}

The present study was financially supported by the County Council of Värmland. Örebro University is gratefully acknowledged for financial support to D.D.

\section{References}

1. Malvezzi M, Bertuccio P, Levi F, La Vecchia C and Negri E: European cancer mortality predictions for the year 2013. Ann Oncol 24: 792-800, 2013.

2. Rutkowski MR, Stephen TL and Conejo-Garcia JR: Anti-tumor immunity: myeloid leukocytes control the immune landscape. Cell Immunol 278: 21-26, 2012.

3. Schiavoni G, Gabriele L and Mattei F: The tumor microenvironment: a pitch for multiple players. Front Oncol 3: 90, 2013

4. Mantovani A: Cancer: inflaming metastasis. Nature 457: 36-37, 2009.

5. McLean MH, Murray GI, Stewart KN, et al: The inflammatory microenvironment in colorectal neoplasia. PLoS One 6: e15366, 2011.

6. Zhou Q, Peng RQ, Wu XJ, et al: The density of macrophages in the invasive front is inversely correlated to liver metastasis in colon cancer. J Transl Med 8: 13, 2010.

7. Forssell J, Oberg A, Henriksson ML, Stenling R, Jung A and Palmqvist R: High macrophage infiltration along the tumor front correlates with improved survival in colon cancer. Clin Cancer Res 13: 1472-1479, 2007.

8. Mosser DM and Edwards JP: Exploring the full spectrum of macrophage activation. Nat Rev Immunol 8: 958-969, 2008

9. Mantovani A, Sica A, Sozzani S, Allavena P, Vecchi A and Locati M: The chemokine system in diverse forms of macrophage activation and polarization. Trends Immunol 25: 677-686, 2004.

10. Biswas SK, Allavena P and Mantovani A: Tumor-associated macrophages: functional diversity, clinical significance, and open questions. Semin Immunopathol 35: 585-600, 2013.

11. Ye Y, Huang X, Zhang Y, et al: Calcium influx blocked by SK\&F 96365 modulates the LPS plus IFN- $\gamma$-induced inflammatory response in murine peritoneal macrophages. Int Immunopharmacol 12: 384-393, 2012.

12. Weisser SB, McLarren KW, Kuroda E and Sly LM: Generation and characterization of murine alternatively activated macrophages. Methods Mol Biol 946: 225-239, 2013.

13. Chen PC, Cheng HC, Wang J, et al: Prostate cancer-derived $\mathrm{CCN} 3$ induces M2 macrophage infiltration and contributes to angiogenesis in prostate cancer microenvironment. Oncotarget 5: 1595-1608, 2014

14. Xiao X, Gaffar I, Guo P, et al: M2 macrophages promote beta-cell proliferation by up-regulation of SMAD7. Proc Natl Acad Sci USA 111: E1211-E1220, 2014.

15. Edin S, Wikberg ML, Dahlin AM, et al: The distribution of macrophages with a M1 or M2 phenotype in relation to prognosis and the molecular characteristics of colorectal cancer. PLoS One 7: e47045, 2012.

16. Kang JC, Chen JS, Lee CH, Chang JJ and Shieh YS: Intratumoral macrophage counts correlate with tumor progression in colorectal cancer. J Surg Oncol 102: 242-248, 2010.

17. Pancione M, Forte N, Sabatino L, et al: Reduced beta-catenin and peroxisome proliferator-activated receptor-gamma expression levels are associated with colorectal cancer metastatic progression: correlation with tumor-associated macrophages, cyclooxygenase 2, and patient outcome. Hum Pathol 40: 714-725, 2009.

18. Bailey C, Negus R, Morris A, et al: Chemokine expression is associated with the accumulation of tumour associated macrophages (TAMs) and progression in human colorectal cancer. Clin Exp Metastasis 24: 121-130, 2007. 
19. Cui YL, Li HK, Zhou HY, Zhang T and Li Q: Correlations of tumor-associated macrophage subtypes with liver metastases of colorectal cancer. Asian Pac J Cancer Prev 14: 1003-1007, 2013.

20. Andre T, Boni C, Navarro M, et al: Improved overall survival with oxaliplatin, fluorouracil, and leucovorin as adjuvant treatment in stage II or III colon cancer in the MOSAIC trial. J Clin Oncol 27 3109-3116, 2009.

21. Graham JS and Cassidy J: Adjuvant therapy in colon cancer. Expert Rev Anticancer Ther 12: 99-109, 2012.

22. McDonald GT, Sullivan R, Paré GC and Graham CH: Inhibition of phosphatidylinositol 3-kinase promotes tumor cell resistance to chemotherapeutic agents via a mechanism involving delay in cell cycle progression. Exp Cell Res 316: 3197-3206, 2010.

23. Miyazaki K, Shibahara T, Sato D, et al: Influence of chemotherapeutic agents and cytokines on the expression of 5-fluorouracil-associated enzymes in human colon cancer cell lines. J Gastroenterol 41: 140-150, 2006.

24. Scartozzi M, Maccaroni E, Giampieri R, et al: 5-Fluorouracil pharmacogenomics: still rocking after all these years? Pharmacogenomics 12: 251-265, 2011.

25. Engström A, Erlandsson A, Delbro D and Wijkander J: Conditioned media from macrophages of M1, but not M2 phenotype, inhibit the proliferation of the colon cancer cell lines HT-29 and CACO-2. Int J Oncol 44: 385-392, 2014.

26. Pfaffl MW, Horgan GW and Dempfle L: Relative expression software tool (REST) for group-wise comparison and statistical analysis of relative expression results in real-time PCR. Nucleic Acids Res 30: e36, 2002.

27. Ruijter JM, Ramakers C, Hoogaars WM, et al: Amplification efficiency: linking baseline and bias in the analysis of quantitative PCR data. Nucleic Acids Res 37: e45, 2009

28. Bossi G, Lapi E, Strano S, Rinaldo C, Blandino G and Sacchi A: Mutant p53 gain of function: reduction of tumor malignancy of human cancer cell lines through abrogation of mutant p53 expression. Oncogene 25: 304-309, 2006.

29. Zhang N, Yin Y, Xu SJ and Chen WS: 5-Fluorouracil: mechanisms of resistance and reversal strategies. Molecules 13: 1551-1569, 2008.

30. Song B, Wang Y, Xi Y, et al: Mechanism of chemoresistance mediated by miR-140 in human osteosarcoma and colon cancer cells. Oncogene 28: 4065-4074, 2009.

31. Harper JW, Elledge SJ, Keyomarsi K, et al: Inhibition of cyclin-dependent kinases by p21. Mol Biol Cell 6: 387-400, 1995.

32. Choi YK, Seo HS, Choi HS, Kim SR, Shin YC and Ko SG Induction of Fas-mediated extrinsic apoptosis, p21WAF1-related G2/M cell cycle arrest and ROS generation by costunolide in estrogen receptor-negative breast cancer cells, MDA-MB-231. Mol Cell Biochem 363: 119-128, 2012.

33. Sturm I, Rau B, Schlag PM, et al: Genetic dissection of apoptosis and cell cycle control in response of colorectal cancer treated with preoperative radiochemotherapy. BMC Cancer 6: 124, 2006.
34. Yoshiba S, Ito D, Nagumo T, Shirota T, Hatori M and Shintani S: Hypoxia induces resistance to 5-fluorouracil in oral cancer cells via G(1) phase cell cycle arrest. Oral Oncol 45: 109-115, 2009.

35. Harper JW, Adami GR, Wei N, Keyomarsi K and Elledge SJ: The $\mathrm{p} 21$ Cdk-interacting protein Cip1 is a potent inhibitor of G1 cyclin-dependent kinases. Cell 75: 805-816, 1993.

36. Rodrigues NR, Rowan A, Smith ME, et al: p53 mutations in colorectal cancer. Proc Natl Acad Sci USA 87: 7555-7559, 1990.

37. Seoane J, Le HV, Shen L, Anderson SA and Massagué J: Integration of Smad and forkhead pathways in the control of neuroepithelial and glioblastoma cell proliferation. Cell 117: 211-223, 2004.

38. Fu G and Peng C: Nodal enhances the activity of FoxO3a and its synergistic interaction with Smads to regulate cyclin G2 transcription in ovarian cancer cells. Oncogene 30: 3953-3966, 2011.

39. Stahl M, Dijkers PF, Kops GJ, et al: The forkhead transcription factor FoxO regulates transcription of p27Kipl and Bim in response to IL-2. J Immunol 168: 5024-5031, 2002.

40. Notas G, Alexaki VI, Kampa M, et al: APRIL binding to BCMA activates a JNK2-FOXO3-GADD45 pathway and induces a G2/M cell growth arrest in liver cells. J Immunol 189: 4748-4758, 2012.

41. Doroshow JH, Multhauf P,Leong L, et al: Prospective randomized comparison of fluorouracil versus fluorouracil and high-dose continuous infusion leucovorin calcium for the treatment of advanced measurable colorectal cancer in patients previously unexposed to chemotherapy. J Clin Oncol 8: 491-501, 1990.

42. Schwartz EL, Baptiste N, Wadler S and Makower D: Thymidine phosphorylase mediates the sensitivity of human colon carcinoma cells to 5-fluorouracil. J Biol Chem 270: 19073-19077, 1995.

43. Pritchard DM, Watson AJ, Potten CS, Jackman AL and Hickman JA: Inhibition by uridine but not thymidine of p53-dependent intestinal apoptosis initiated by 5-fluorouracil: evidence for the involvement of RNA perturbation. Proc Natl Acad Sci USA 94: 1795-1799, 1997.

44. Peters GJ, van Groeningen CJ, Laurensse EJ and Pinedo HM: A comparison of 5-fluorouracil metabolism in human colorectal cancer and colon mucosa. Cancer 68: 1903-1909, 1991.

45. Etienne MC, Chéradame S, Fischel JL, et al: Response to fluorouracil therapy in cancer patients: the role of tumoral dihydropyrimidine dehydrogenase activity. J Clin Oncol 13: 1663-1670, 1995

46. Takagi K, Sowa Y, Cevik OM, Nakanishi R and Sakai T: CDK inhibitor enhances the sensitivity to 5-fluorouracil in colorectal cancer cells. Int J Oncol 32: 1105-1110, 2008.

47. Matsushita S, Ikeda R, Nishizawa Y, et al: The role of thymidine phosphorylase in the induction of early growth response protein-1 and thrombospondin-1 by 5 -fluorouracil in human cancer carcinoma cells. Int J Oncol 36: 1193-1200, 2010. 